\title{
SENSORY OUTCOME AFTER SURGERY FOR INTERMITTENT EXOTROPIA
}

\author{
Farrag, A. ${ }^{1(*)}$, Abdel Badie, M. ${ }^{2}$, \& Saleh, M. ${ }^{3}$ \\ ${ }^{1,2}$ Ophthalmology dept., Faculty of Medicine, AL-Azhar Univ., Assuit, Egypt \\ ${ }^{3}$ Ophthalmology dept, Faculty of Medicine, AL-Azhar Univ. Cairo, Egypt \\ *E-mail: ahmed_alhady2010@yahoo.com
}

\begin{abstract}
Purpose: To investigate whether patients with intermittent exotropia can achieve better postoperative sensory outcome, including improvement in the stereo-acuity and increase in the grade of binocular single vision. Methods: 20 patients diagnosed as intermittent exotropia who underwent surgery, motor changes measured with an alternate cover test and Simultaneous prism and cover test and Assessment of sensory changes by Worth's 4 dot test used to assess fusion for near and far and Stereoacuity measurements performed using global TNO test. Results: Males were 11 in numbers (55\%) while females were 9 (45\%). age ranges from 5-27 years. Range of BCVA was (0.2-1). Mean $+S . D=0.8+0.223 .16$ out of 20 patients achieved orthotropia postoperative, while there was 2 patients with overcorrection and 2 patients with under correction. W4D for far shows significant improvement $p=0.99$ while $W 4 D$ for near shows Insignificant improvement $p=0.223$. TNO stereotest: 16 out of 20 patients could pass the test, only four patients couldn't pass it completely pre-op. Conclusions: Impairment of BSV in (x)t is usually in the form of suppression. The most severe binocular impairment is monofixaton syndrome with loss of all central binocular vision \& development of central scotoma, Stereoacuity is diminished but in a mild form. It can return to normal levels in many patients by appropriate surgery.
\end{abstract}

Keywords: Intermittent exotropia, Binocular vision, Stereopsis, Amblyopia

\section{Introduction}

Intermittent exotropia $\mathrm{X}(\mathrm{T})$ is a relatively common form of strabismus in about $25 \%$ of all cases of strabismus and in $1 \%$ of the general population [1]. It is a large exophoria that intermittently breaks down to an exotropia. Occluding one eye breaks fusion and will manifest the exotropia. The exotropia is typically manifest when the patient is fatigued, daydreaming or ill [2]. Approximately $80 \%$ of intermittent exotropia patients will show progressive loss of fusion control and an increase in the exotropia over several months to years. Adult patients can have extremely large deviation [3]. Treatment of intermittent XT is aimed to reducing episodes of manifest 
exotropia, prevention of amblyopia, facilitating sensory fusion and achieving constant binocular alignment and normal stereoacuity [4]. Treatment approaches include: over minus lenses; orthoptic training to improve range of sensory fusion; occlusion therapy (antisuppression treatment); Horizontal rectus surgery with/without oblique muscle surgery for $\mathrm{A}$ or $\mathrm{V}$ pattern

\section{Patients and Methods}

This prospective clinical study included 20 patients diagnosed as intermittent exotropia who had been recruited from the Ophthalmology Department at Alazhar University Hospital, Assuit from October 2015 to September 2016. An informed written consent was obtained from each patient before enrollment in the study. Al-Azhar university Institutional Ethics Committee approval was obtained. Exclusion criteria includes Patients with history of previous eye muscle surgery, Patients with vertical muscle dysfunction, Patients with neurological disorders and Patients with nystagmus. Preoperative examination involved a detailed history, assessment of visual acuity, anterior segment examination, ocular media examination, the amount of exodeviation, Duction and Version, Cycloplejic refraction, and fundus examination. The ocular deviation was

\section{Statistical analysis}

Categorical variables were described by number and percent $(\mathrm{N}, \%)$ and continuous variables were described by range, mean and standard deviation (Mean $+\mathrm{SD}$ ), median and IQ). For parametric data; one way ANOVA test, and pairedsamples t-test were used to compare

\section{Results}

Of the 20 patients in this study, Males were 11 in numbers $(55 \%)$ while females were 9 (45\%). age ranges from (5-27 years, mean $=11.45$, standard deviation $=7.72$ ) . Patients age $<15=16$ patients $(80 \%)$, and $>15=4$ patients $(20 \%)$. Of total sample exotropia [5]. In this study we investigate whether patients with intermittent exotropia can achieve better post-operative sensory outcome, including improvement in the stereo-acuity and increase in the grade of binocular single vision and whether preoperative sensory status can influence the decision of surgery.

measured with an alternate cover test and Simultaneous prism and cover test at $6 \mathrm{~m}$ and $33 \mathrm{~cm}$ to an accommodative target. Assessment of sensory changes by Worth's 4 dot test used to assess fusion (central and peripheral) for near and far. Stereo acuity measurements performed using global TNO test charts (stereoscopic acuity test of the Netherlands Organisation for Applied Scientific Research, Lameris Ootech BV, Nieuwegein, The Netherlands). Bilateral Lateral Rectus Recession is the preferred surgery. In patients with amblyopia $20 / 50$ or worse, a recession/resection procedure on the amblyopic eye is preferred avoiding surgery on the "good" eye. Patients were examined on postoperative day 1, week 1, week 6 and at 6 months. Sensory and motor outcome measures determined 6 months after surgery.

between data. For non-parametric data; Wilcoxon test was used to compare between data. For correlation; we used Pearson correlation. The significance level was set at $\mathrm{P}<0.05$. Statistical analysis was performed with IBM SPSS Statistics Version 20 for Windows.

of $25 \mathrm{X}(\mathrm{T})$ patients, 5 were lost for follow up. Total numbers of eyes were 40 eyes of 20 patients. Range of BCVA was (0.2-1). Mean + S.D $=0.8+0.223$. They were categorized into 3 groups: *) 0.2-0.5 (7 eyes, $17.5 \%$ ). *) 0.6-0.8 (16 eyes, $40 \%$ ). 
*) 0.9-1 (17 eyes, 42.5\%).5 patients had amblyopia worth than $20 / 30$ (25\%). Two of them had amblyopia in both eyes, while the other 3 had amblyopia in one eye only. One female patient suffers from high myopia of spherical equivalent about (- 10 right eye, -11 left eye). None had significant hypermetropia and 3 patients only suffered from significant astigmatism. Most common type of surgery was bilateral lateral rectus recession (19 times) versus one surgery of unilateral recession resection plus unilateral lateral rectus recession to treat large angle $\mathrm{X}(\mathrm{T})$ of $60 \mathrm{PD} .16$ out of 20 patients achieved orthotropia postoperative, while There was 2 patients with overcorrection; esotropia of 20,25 PD and they had a successful second surgery. The other 2 patients with under correction: one had 18 PD of residual X(T), he improved to orthotropia after the $2^{\text {nd }}$ operation. The other one had 14 PD of exophoria, but she didn't go to surgery as she was satisfied with the results, fig. (1).

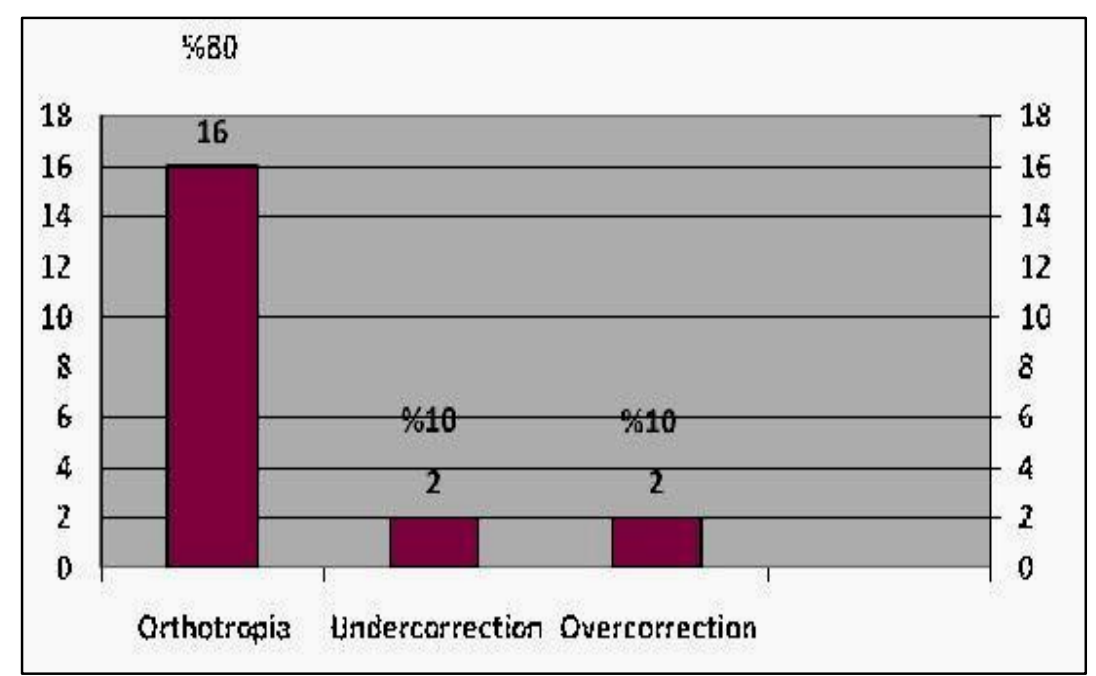

Figure (1) Distribution of the studied patients regarding the outcome after the $1^{\text {st }}$ surgery.

Mean exotropic deviation for far preoperative was $(34 \mathrm{PD}+13.338)$, while postoperative was $(0.55+9.72)$. Mean deviation for near preoperative was $(32.65+$ 13.433), while postoperative was $(1.5+$
5.539). Numbers of patients suffered from small - moderate $\mathrm{X}(\mathrm{T})$ of less than $35 \mathrm{PD}$ $\mathrm{X}(\mathrm{T})$ were 14 , and 6 suffered from large $\mathrm{X}(\mathrm{T})$ of more than $35 \mathrm{pd}$, fig. (2).

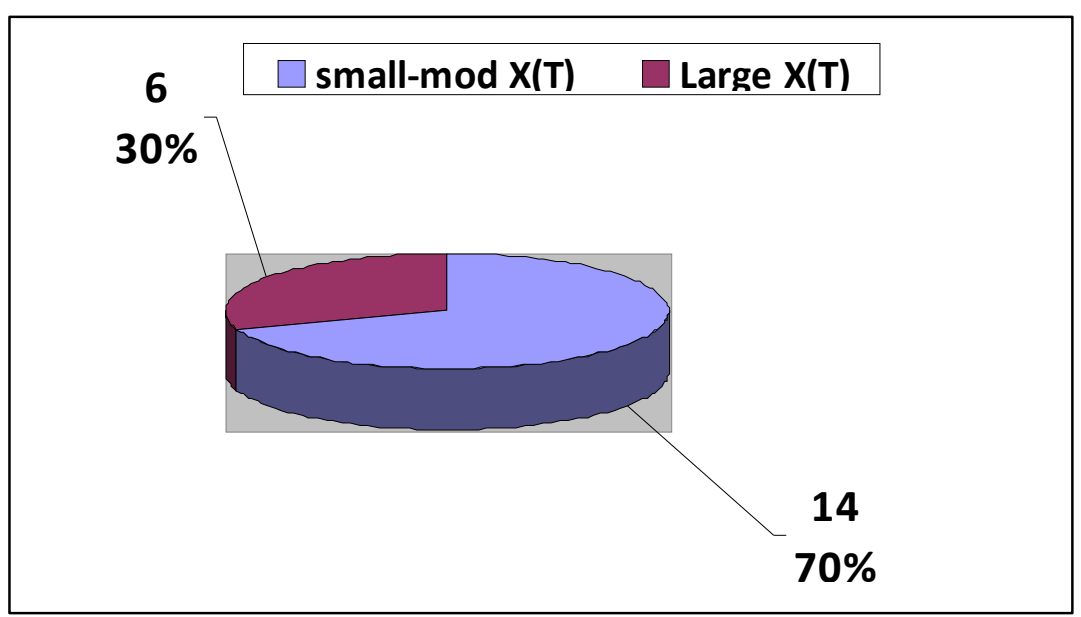

Figure (2) Classification of Angle deviation for far. 
W4D for far: 13 (65\%) patients had fusion pre op. and $6(30 \%)$ had suppression, only one had diplopia. Patient suffered from diplopia improved to fusion post-op. 2 patients with suppression improved to fusion post-op. while 3 patients with fusion pre-op. changed to suppression post-op. (significant improvement $\mathrm{p}=0.99$ ), fig. (3). W4D for near: 15 patients $(75 \%)$ had fusion pre op, 4 (20\%) had suppression and one (5\%) only had diplopia. Post op. 18 had fusion and 2 only had suppression (Insignificant improvement $\mathrm{p}=0.223$ ), fig.
(4). TNO stereo test: 16 out of 20 patients could pass the test, only four patients couldn't pass it completely preop. and scored Nil, one of them only pass it post-op. and reached 480 arcsec. The median was 240 (range $=60-\mathrm{Nil})$ arcsec pre-op, it improved significantly post op to 60 (30-Nil) arcsec. $(\mathrm{P}=0.0025)$. The 16 patients only with measurable stereoacuity pre-op. were plotted on a Barr Chart to compare pre and post-op. measurements, fig. (5).

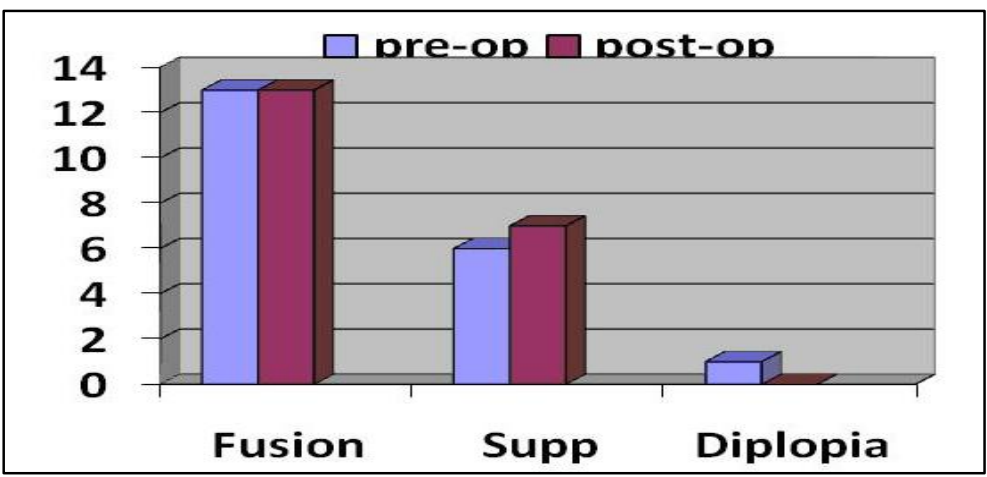

Figure (3) Comparison between W4d-far pre-op. and 6 months post-op.

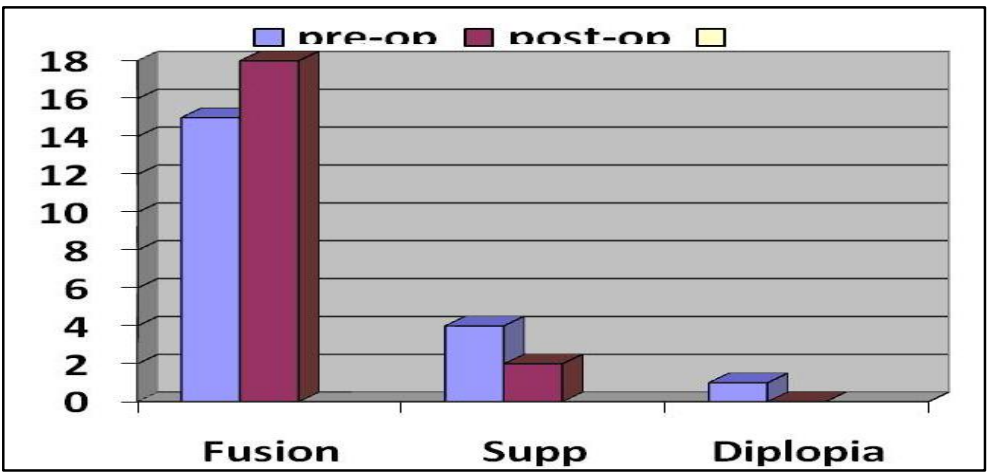

Figure (4) Comparison between W4D-near pre-op. and 6 months post-op.

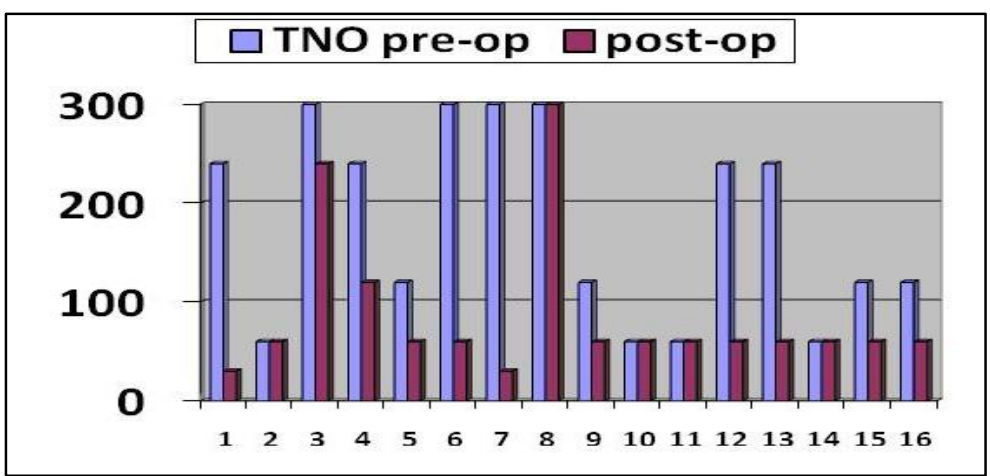

Figure (5) Comparison between TNO Stereotest pre-op. and post-op. 
4 patients (20\%) had fine stereopsis pre-op. and increased to 13 (65\%). 9 patients $(45 \%)$ had intermediate stereopsis between (>60 - 480 arcsecs), 7 of them improved to fine stereopsis. 3 patients (15\%) had gross stereopsis pre-op, one of them improved to fine stereopsis post-op (30 arcsecs) and another one improved to intermediate stereopsis (240 arcsecs), the later of them has high myopia (around -11 D OU) and amblyopia, while the first one had large angle $\mathrm{X}(\mathrm{T})$ of 80 PD. 4 patients $(20 \%)$ scored nil pre \& post-op, these results are listed in tab. (1) \& show in fig. (5).

Table (1) Classification according to level of stereopsis in TNO stereotest pre-op. and post-op.

\begin{tabular}{|l|c|c|}
\hline & Preoperative & Postoperative \\
\hline$<60$ & $4(20 \%)$ & $12(60 \%)$ \\
$>\mathbf{6 0}$ & $9(45 \%)$ & $3(15 \%)$ \\
Gross & $3(15 \%)$ & $1(5 \%)$ \\
Nil & $4(20 \%)$ & $4(20 \%)$ \\
\hline Total & 20 & 20 \\
\hline
\end{tabular}

\begin{tabular}{|c|c|c|}
\hline & Pre-op. & Post-op. \\
\hline Case no. 1 & & \\
\hline Case no. 2 & & \\
\hline Case no. 3 & & \\
\hline Case no. 4 & & \\
\hline
\end{tabular}

Figure (5) Shows 4 cases as examples for surgical correction before and after surgery

\section{Discussion}

This study was done to detect any improvement or changes in binocular vision in general \& stereoacuity in special after the operation for intermittent exotropia. Most of them were children $\leq 15=16$ patients $(80 \%)$, and $>15=4$ patients $(20$ 
$\%)$. Age ranges from $(5-34 \mathrm{yrs}$, mean $=$ 11.45, Standard deviation $(\mathrm{SD})=7.72$ ) Of a total number of $25 \mathrm{X}(\mathrm{T})$ patients, 13 were females $(52 \%) \& 12$ were males $(48 \%)$. This agrees with most studies of female predominance $(51.6 \%, 53.8 \%$ and $60.3 \%$ ) [6-8]. But 20 only continued the study as the rest were lost for follow-up. 11 were males $(55 \%)$ and 9 were females $(45 \%)$. One study showed that females are nearly twice that of males [9]. Other studies showed that males are more (57.6\%, 51.8\% and 52.4\%) [10-12]. Mean BCVA for all eyes was $0.8 \pm 0.223$. This was very similar to another study formed by Yang at al. which was 0.78 [7]. In general W4D test for near showed that many patients could improve. But W4D for far didn't show improvement for patients, some of them improved to fusion but others worsened to suppression which couldn't be explained, the patients who worsened had excellent near steoracuity of less than 60 arcsecs on TNO \& excellent surgical results of orthotropia for far. But in general this proves that binocular function is usually worsened for distance more than for near in patient with $\mathrm{X}(\mathrm{T})$. This was also proved by another study by Yildrim et al. where he noticed that $35 \%$ of patients with $\mathrm{X}(\mathrm{T})$ has central suppression and $65 \%$ had central fusion( exactly the same as the results of this study that showed that $65 \%$ had central fusion by W4D for far) but he used more sensitive test than W4D, this test is called (distance alternate-letter suppression test) in addition to W4D. While on W4d test he found that all $\mathrm{X}(\mathrm{T})$ patients $(\mathrm{n}=26)$ had fusion for near \& far [13]. Also another study by Cooper about suppression \& ARC in patients with $\mathrm{X}(\mathrm{T})$, he found that $\mathrm{X}(\mathrm{T})$ patients could change from ARC/NRC to suppre-ssion according to the type of test used, intensity, color \& size of targets [14]. A study was held in Korea yields results close to our study, they used also flashlight W4d to test near \& far fusion. Jung studied 398 patients with $\mathrm{X}(\mathrm{T})$, most of them were children (360 children) and few adults (38 adult). He found that $50.3 \%$ has fusion on W4D for far, compared to our results of $65 \%$. And $74.9 \%$ on W4D for near compared to this study's results of 75\% [11]. In TNO Stereotest: The median was 240 (range $=60-\mathrm{Nil}$ ) arcsecs pre-op, it improved post-op to 60 (30-Nil) arcsecs (significant improvement, $\mathrm{p}=0.0025$ ). 11 (55\%) improved, none worsened and 9 (45\%) no change. 4 patients $(20 \%)$ had fine stereopsis pre-op and increased to 12 (60\%). 9 patients $(45 \%)$ had intermediate stereopsis between (>60 - 480 arcsecs), 7 of them improved to fine stereopsis. 3 patients $(15 \%)$ had gross stereopsis pre-op, one of them improved to fine stereopsis post-op (30 arcsecs) and another one improved to intermediate stereopsis (240 arcsecs), both patients were females with poor control, in addition that the later of them has high myopia (around -11 D OA) and amblyopia, while the first one had large angle $\mathrm{X}(\mathrm{T})$ of 80 PD. 4 patients (20\%) scored nil pre \& post-op. A study by Yildrim et al. on $26 \mathrm{X}(\mathrm{T})$ patients and 112 normal subjects, all were over than 5 years, they found that median stereoacuity on TNO was 60 arcsecs pre \& post-op, and also for normal subjects. This agrees with this study of post-op results but disagrees with pre-op results. In Yildrim study $100 \%$ of patients had stereopsis using TNO stereotest. They found also that 
distance stereoacuity is mainly deteriorated in patients with $\mathrm{X}(\mathrm{T})$ than near stereoacuity [13]. Another study by Yildirim and Altinsoy was done on $71 \mathrm{X}(\mathrm{T})$ patients (5-57 years old, $75 \%$ younger than 20 yrs old) \& 112 normal subjects as a control, they found that 60 arcsecs was the median for $\mathrm{X}(\mathrm{T})$ patients \& also for controls using TNO stereotest [15].

\section{Conclusion}

Binocular vision in $X(T)$ is impaired. Impairment is usually in the form of suppression. The most severe binocular impairment is monofixaton syndrome with loss of all central binocular vision \& development of central scotoma, which once happened, will probably stay forever even after surgery. Stereoacuity is diminished but in a mild form. It can return to normal levels in many patients by appropriate surgery. In case there is measurable stereoacuity, it can return to within normal levels at any age post-operative.

\section{References}

1. Chef, E., Remaley, N., Tamboli, A., et al. Risk factors for esotropia and exotropia. Arch Ophthalmol. 1994; 112: 49-1355.

2. Rutstien, R. \& Daum K. Anomalis of binocular vision: Diagnosis and management, $1^{\text {st }}$ ed., Mosby, USA.

3. Hatt, S., Mohney, B., Leske, D., et al. Variability of control of intermittent exotropia. Ophthalmology. 2008; 115: 371-376.

4. Figueira, E. \& Hing, S. Intermittent exotropia: Comparison of treatments. Clin Exp Ophthalmol. 2006; 34 (3): 245-251.

5. Tyler, C. Binocular Vision. Foundation Vol. 2, Ch. 24. In: Tasman, W., (ed.) Duane's Clinical Ophthalmology. 2005, Lippincott Williams \& Wilkins, New York.

6. Sharma, P., Saxena, R., Narvekar, M., et al. Evaluation of distance and near stereoacuity and fusional vergence in intermittent exotropia. Indian J Ophthalmol 2008; 56: 121-125.

7. Yang, C., Shen, Y., Gu, Y., et al. Clinical investigation of surgery for intermittent exotropia. J Zhejiang Univ Sci B. 2008; 9 (6): 470-473.

8. Rutstein, R. \& Corliss, D. The clinical course of intermittent exotropia. Optom Vis Sci. 2003; 80 (9): 4-649.

9. Nusz, K., Mohney, B., Diehl, N. Female predominance in intermittent exotropia. Am J Ophthalmol. 2005; 140 (3): $546-547$.

10. Kim, C. \& Hwang, J. 'Largest angle to target' in surgery for intermittent exotropia. Eye. 2005; 19(6):637-642.

11. Jung, J. \& Lee, S. A comparison of the clinical characteristics of intermittent exotropia in children and adults. Korean J Ophthalmol. 2010; 24 (2): 96-100.

12. Wu, H., Sun, J., Xia, X., et al. Binocular status after surgery for constant and intermittent exotropia. Am J Ophthalmol 2006; 142 (5): 822-826

13. Yildirim, C., Mutlu, F., Chen, Y., et al. Assessment of central peripheral fusion and near and distance stereoacuity in intermittent exotropic patients before and after strabismus surgery. Am J Ophthalmol. 1999; 128 (2): 222-230.

14. Cooper, J. \& Record, C. Suppression and retinal correspondence in intermittent exotropia. Br J Ophthalmol 1986; 70 (9): 673-676.

15. Yildirim, C. \& Altinsoy, H. Distance alternate-letter suppression test for objective assessment of sensorial status in intermittent exotropia. Eur J Ophthalmol. 2000; 10 (1):4-10. 